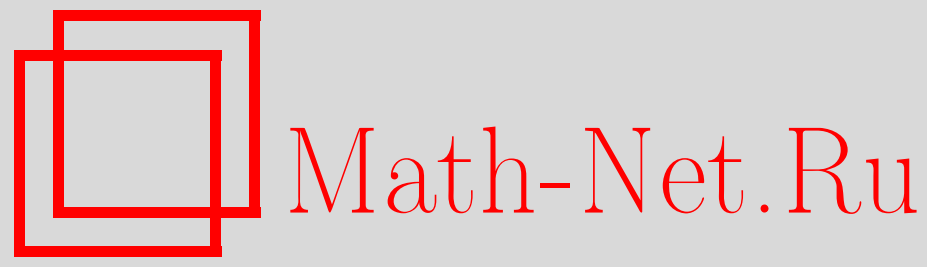

В. А. Клепцын, Д. А. Филимонов, Структура групп диффеоморфизмов окружности со свойством неподвижности нерастяжимых точек, Функи. анализ и его прил., 2012, том 46, выпуск 3, 38-61

DOI: https://doi.org/10.4213/faa3082

Использование Общероссийского математического портала MathNet.Ru подразумевает, что вы прочитали и согласны с пользовательским соглашением

http://www . mathnet.ru/rus/agreement

Параметры загрузки:

IP : 35.173 .219 .12

26 апреля 2023 г., 13:55:58

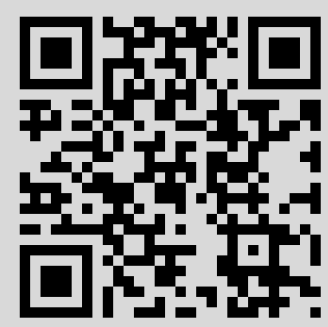




\title{
Структура групп диффеоморфизмов окружности со свойством неподвижности нерастяжимых точек*
}

\author{
(c) 2012. В. А. Клепцын, Д. А. Филимонов
}

Работа посвящена исследованию групп диффеоморфизмов окружности со свойством неподвижности нерастяжимых точек. Это свойство обобщает свойство локальной растяжимости, и на текущий момент не известно примеров минимальных действий конечно порожденных групп $C^{2}$-диффеоморфизмами окружности, которые бы этим свойством не обладали.

Оказывается, что в предположении, что диффеоморфизмы обладают указанным свойством, и при наличии хотя бы одной нерастяжимой точки, действие допускает достаточно жесткое описание. В частности, для него доказывается существование разбиения Маркова, а по своей структуре такое действие оказывается близким к действию группы Томпсона.

\section{$\S 1$. Введение}

1.1. Предпосылки: гипотеза об эргодичности. Одним из известных вопросов теории динамических систем является следующая

Гипотеза 1. Рассмотрим конечно порожденную группу $G \subset \operatorname{Diff}^{2}\left(S^{1}\right)$. Eсли ее действие минимально (т.е. любая G-орбита плотна), то оно эргодично относительно меры Лебега (m.е. любое измеримое инвариантное множество имеет меру 0 или 1).

Эта гипотеза была сформулирована в 80-х годах XX века многими авторами, включая Д. Салливана, Ж. Эктора и Э. Жиса. Отметим, что даже для (послужившего мотивацией для гипотезы в целом) случая одного диффеоморфизма окружности $(G \simeq \mathbb{Z})$ это утверждение не является очевидным следствием классификационной теоремы Пуанкаре. Дело в том, что сопряжение между минимальным диффеоморфизмом и соответствующим иррациональным поворотом может не быть абсолютно непрерывным, поэтому эргодичность поворота не влечет за собой эргодичность в смысле меры Лебега исходного отображения.

Тем не менее с помощью более тонких рассуждений в случае одного диффеоморфизма гипотеза была доказана - одновременно и независимо - А. Б. Катком (см. изложение в [12]) и М. Эрманом [9].

Для случая более богатой (не сохраняющей никакую меру) динамики основной идеей, лежащей в основе доказательств эргодичности, является восходящая к Д. Салливану (см. [17, следствие 3], [15, предложение 4.3]) идея экспоненциального растяжения (и, более общо, растяжения с контролем искажения):

*Работа выполнена при частичной поддержке русско-французской программы «Cooperation network in mathematics», гранта РФФИ 10-01-00739-а и гранта РФФИ-CNRS 10-01-93115-НЦНИЛ_а. 
Теорема (Д. Салливан). Пусть группа $G \subset \operatorname{Diff}^{1+\varepsilon}\left(S^{1}\right)$ действует на окружности минимально и

для любого $x \in S^{1}$ существует $g \in G$, такой, что $g^{\prime}(x)>1$.

Тогда ее действие эргодично относительно мерь Лебега.

Отметим, что препятствием к применению этой техники является наличие нерастяжимых точек:

Определение 1. Точка $x \in S^{1}$ называется нерастяжимой (для действия группы $G)$, если

$$
\left|g^{\prime}(x)\right| \leqslant 1 \text { для любого } g \in G .
$$

(Такое название, конечно, является некоторой вольностью речи - было бы корректнее говорить о точках с нерастяжимым касательным пространством, - однако эта вольность позволяет сократить изложение.)

Множество нерастяжимых точек мы будем обозначать через $\mathrm{NE}=\mathrm{NE}(G)$.

Для простоты мы в дальнейшем ограничимся случаем сохраняющих ориентацию диффеоморфизмов, поскольку такие диффеоморфизмы образуют подгруппу индекса не больше 2 , а переход к подгруппе конечного индекса сохраняет минимальность действия на окружности.

Наличие нерастяжимых точек не противоречит минимальности действия группы (даже аналитических) диффеоморфизмов: примерами служат (см. [4]) стандартное действие $P S L_{2}(\mathbb{Z})$ и (для гладкого случая) гладкая минимальная реализация Жиса-Сержиеску [6] группы Томпсона.

Однако все известные на текущий момент примеры минимальных действий с нерастяжимыми точками отличаются от этих двух незначительными модификациями.

В частности, они все обладают следующим свойством: нерастяжимые точки являются изолированными неподвижными для некоторых элементов группы. Более точно, они обладают свойством $(\star)$ из следующего определения.

Определение 2 [4]. Конечно порожденная группа $G \subset \operatorname{Diff}^{2}\left(S^{1}\right)$ обладает свойством $(\star)$, если ее действие минимально и для всякой точки $x \in \operatorname{NE}(G)$ найдутся $g_{+}, g_{-} \in G$, такие, что $g_{+}(x)=g_{-}(x)=x$ и $x$ является изолированной справа (соответственно слева) точкой в $\operatorname{Fix}\left(g_{+}\right)$(соответственно в $\left.\operatorname{Fix}\left(g_{-}\right)\right)$.

Это мотивировало исследования обладающих этим свойством групп в работе [4].

Оказывается, для таких групп все еще возможно построить процедуру растяжения с контролем искажения (хотя скорость растяжения оказывается уже не экспоненциальной по числу итераций). Как следствие, действие такой группы оказывается эргодичным относительно меры Лебега. Отметим также, что одним из следствий является конечность множества NE.

Более того, оба упомянутых выше примера (группа Томпсона и $P S L_{2}(\mathbb{Z})$ ) обладают рядом других интересных свойств: они порождаются (в определенном смысле) нестрого растягивающей «марковской» динамикой, для них показатель Ляпунова растяжения равен нулю. Естественный возникающий в связи с этим вопрос - а любая ли группа с непустым множеством нерастяжимых точек, обладающая свойством $(\star)$, обладает такими свойствами? И можно ли такие действия классифицировать? 
1.2. Формулировка результатов. Настоящая работа посвящена ответам на эти вопросы - исследованию групп со свойством ( $)$ и непустым множеством нерастяжимых точек. Для краткости мы будем называть такие группы $N$-группами. А именно, мы докажем следующее утверждение:

Теорема 1 (марковская нестрого растягивающая динамика). Пусть $G-$ конечно порожденная $N$-группа $C^{2}$-диффеоморфизмов окружности. Тогда для нее существуют разбиение окружности на интерваль $\left\{I_{1}, \ldots, I_{k}, I_{1}^{+}, I_{1}^{-}, \ldots, I_{l}^{+}\right.$, $\left.I_{l}^{-}\right\}=\mathcal{I}$ и соответствуюшие этим интервалам отображсения

$$
g_{1}, \ldots, g_{k}, g_{1}^{+}, g_{1}^{-}, \ldots, g_{l}^{+}, g_{l}^{-} \in G,
$$

такие, что:

(i) все образы $g_{i}^{ \pm}\left(I_{i}^{ \pm}\right), g_{j}\left(I_{j}\right)$ представляются как объединения интервалов u3 $\mathcal{I}$;

(ii) существует $\lambda_{0}>1$, такое, что $g_{j}^{\prime}(x) \geqslant \lambda_{0}$ для любого $j$ и любого $x \in I_{j}$;

(iii) интервалы $I_{i}^{+}$и $I_{i}^{-}$примыкают соответственно справа и слева к нерастяжимой точке $x_{i}^{*} \in \mathrm{NE}$, причем $x_{i}^{*}$ - неподвижная топологически отталкивающая точка ограничения на интервал $I_{i}^{+}$(соответственно $I_{i}^{-}$) отображсения $g_{i}^{+}$(соответственно $\left.g_{i}^{-}\right)$, не имеющего на этом интервале других неподвижних точек; кроме того, $g_{i}^{ \pm}\left(I_{i}^{ \pm}\right) \backslash I_{i}^{ \pm} \subset \bigcup_{j} I_{j}$.

(iv) $\left(g_{j(x)} \circ\left(g_{i}^{ \pm}\right)^{k_{i}^{ \pm}(x)}\right)^{\prime}(x) \geqslant 2$ для любого $i$ июбого $x \in I_{i}^{ \pm}$, где $k_{i}^{ \pm}(x):=$ $\min \left\{k \in \mathbb{N} \mid\left(g_{i}^{ \pm}\right)^{k}(x) \notin I_{i}^{ \pm}\right\}$, a $j(x)$ определяется условием $\left(g_{i}^{ \pm}\right)^{k_{i}^{ \pm}(x)}(x) \in I_{j(x)}$.

Замечание 1. Для удобства мы будем иногда обозначать через $g_{I}$ отображение, соответствующее интервалу $I$ разбиения $\mathcal{I}$. Кроме того, нам будет удобно зафиксировать константу $\lambda_{0}$ из п. (ii) и положить $\lambda:=\min \left\{\lambda_{0}, 2\right\}>1$; эта новая константа одновременно оценивает снизу производную отображения $g_{j}$ в любой точке $x$ соответствующего интервала $I_{j}$ и производную композиции $g_{j(x)} \circ\left(g_{i}^{ \pm}\right)^{k_{i}^{ \pm}(x)}$ в любой точке $x \in I_{i}^{ \pm}$.

Теорема 1 утверждает существование для конечно порожденных N-групп $C^{2}$-диффеоморфизмов марковских разбиений; далее мы воспользуемся существованием такого разбиения для более точного описания структуры действия. Стоит отметить, что динамика псевдогрупп, допускающих марковское разбиение на минимальном множестве, как и вопросы существования такого разбиения, уже исследовались в литературе; см., например, [1]-[3].

Пусть $G$ есть N-группа. Выберем и зафиксируем разбиение окружности на интервалы с соответствующими им отображениями, $\left\{\left(I, g_{I}\right) \mid I \in \mathcal{I}\right\}$, существование которого устанавливается в теореме 1. Это разбиение задает процедуру растяжения, которую можно применять к точкам и к достаточно малыми интервалам:

Определение 3. Последовательность растяюения интервала $J \in S^{1}-$ это конечная последовательность интервалов $J^{(n)}$, построенная по правилу

- $J^{(0)}=J$;

- $J^{(n)}=g_{I_{(n)}}\left(J^{(n-1)}\right)$, если $J^{(n-1)} \subset I_{(n)} \in \mathcal{I}$; если же такого интервала $I_{(n)}$ не найдется, то последовательность останавливается на $J^{(n-1)}$.

При этом композиции $G_{n, J}:=g_{I_{(n)}} \circ \cdots \circ g_{I_{(1)}}$ называются растягивающими композициями для интервала $J$. Обозначим также через $G_{J}$ максимальную 
растягивающую композицию, т. е. композицию всех последовательных отображений $g_{I_{(k)}}$ с условием остановки, описанным выше.

Наконец, такая же (только бесконечная) процедура растяжения может быть определена и для точек. Для точки, принадлежащей орбите нерастяжимой точки, при этом необходимо уточнить, растягивается ли ее правая или левая окрестность; в этих случаях мы будем обозначать растягивающие композиции соответственно через $G_{n, x}^{+}$и $G_{n, x}^{-}$.

Использовав эту процедуру увеличения как своеобразный «микроскоп», мы увидим, что все отображения из группы $G$, рассматриваемые под достаточным увеличением, построены из конечного числа элементарных «кирпичиков»:

Теорема 2 (о структуре). Пусть группа $G$ такая же, как в теореме $1, u$ интервалы и отображения $\left(I_{j}, g_{j}\right),\left(I_{i}^{ \pm}, g_{i}^{ \pm}\right)$из заключения этой теоремъ въбраны и зафиксированы. Тогда найдется конечное число интервалов $L_{1}, \ldots, L_{N}$, $L_{1}^{\prime}, \ldots, L_{N}^{\prime}$ и отображений $h_{i}: L_{i} \rightarrow L_{i}^{\prime}$, таких, что любое отображсение $g \in G$ может быть представлено следующим образом:

- имеются два разбиения окружности в обвединение интервалов, зависящие от выбора $g$ :

$$
S^{1}=J_{1} \cup \cdots \cup J_{m}=g\left(J_{1}\right) \cup \cdots \cup g\left(J_{m}\right) ;
$$

- для каждого $p=1, \ldots, m$ в последовательности растяэсения интервала $J_{p}$ встречается некоторый интервал $L_{i_{p}}$, а в последовательности растяэения интервала $g\left(J_{p}\right)$ - соответствующий ему интервал $L_{i_{p}}^{\prime} ;$ иными словами, для некоторых $n, n^{\prime}$

$$
G_{n, J_{p}}\left(J_{p}\right)=L_{i_{p}}, \quad G_{n^{\prime}, g\left(J_{p}\right)}\left(g\left(J_{p}\right)\right)=L_{i_{p}}^{\prime} ;
$$

- отображение g под таким увеличением оказывается соответствующим отображсением $h_{i_{p}}$ :

$$
\left.g\right|_{J_{p}}=G_{n^{\prime}, g\left(J_{p}\right)}^{-1} \circ h_{i_{p}} \circ G_{n, J_{p}} .
$$

Более того, разбиение $S^{1}=\bigcup J_{i}$ можно выбрать одним и тем же для любого конечного набора отображсний из $G$.

Таким образом, мы видим, что действие группы $G$ по построению напоминает действие группы Томпсона.

Напомним, что группа Томпсона - группа кусочно аффинных гомеоморфизмов окружности, сохраняющих множество двоично-рациональных точек, причем концы отрезков аффинности также двоично-рациональны; с этой группой связана, например, известная проблема ее аменабельности. Эквивалентным образом, эта группа может быть определена как состоящая из гомеоморфизмов, кусочно являющихся композициями итераций отображения удвоения $x \mapsto 2 x$ и ветвей, обратных к его степеням, причем точки «склеивания» участков таких представлений должны быть двоично-рациональными. (Отметим, что с помощью этого представления Жии и Сержиеску в работе [6] доказали рациональность числа вращения любого гомеоморфизма из группы Томпсона.)

В упомянутой выше аналогии с группой Томпсона отображение удвоения $x \mapsto 2 x$ заменяется на (лишь кусочно непрерывное) отображение $R$, заданное формулой $R(x)=g_{I}(x), x \in I$. При этом между применением прямых итераций $R^{m}$ и ветвей обратных итераций $R^{-n}$ заключение теоремы 2 предписывает применение одного из конечного множества отображений $h_{i}$. Хотя это описание 
и отличается от канонического описания группы Томпсона, отличие не столь сильно, как может показаться: так, обычную группу Томпсона можно задавать также и с помощью отображения учетверения $x \mapsto 4 x$, между применением его степени и ветви обратного к его степени применяя либо тождественное отображение, либо ветвь $x \mapsto x / 2$.

Рассмотрим теперь заданное отображением $R$ отношение эквивалентности $\mathcal{R}$. Классы эквивалентности такого отношения в силу определения являются подмножествами соответствующих $G$-орбит. Более того, для всех точек, кроме не более чем счетного множества, их классы можно рассматривать (соединяя точку с ее $R$-образом) как деревья с выделенным направлением на бесконечности (соответствующим итерированию отображения $R$ ). Интересно отметить, что имеет место

Следствие 1. Всякая $G$-орбита разбивается на не более чем $N$ классов $\mathcal{R}$-эквивалентности.

Мы упомянем здесь также еще два результата, относящиеся к исследованию показателей Ляпунова, которые могут быть выведены из теорем 1 и 2. Их доказательства публикуются в отдельной работе (см. [13]).

А именно: итерации отображения $R$ - это, в определенном смысле, «жадный» алгоритм растяжения окрестности заданной точки. Оказывается, что поскольку $\mathrm{NE} \neq \varnothing$, из наличия параболических точек следует

Теорема 3. Для почти любой по мере Лебега точки $x \in S^{1}$ показатель Ляпунова отображения $R$ в ней равен нулю, $\lambda(x, R)=0$.

Напомним следующее определение.

Определение 4. Пусть $G \subset \operatorname{Diff}^{2}\left(S^{1}\right)$ - конечно порожденная группа и $\mathcal{F}=\mathcal{F}^{-1}$ - конечная симметричная система ее образующих. Тогда величина

$$
\lambda_{\exp }(x ; \mathcal{F})=\limsup _{n \rightarrow \infty} \frac{1}{n} \max _{f_{1}, \ldots, f_{n} \in \mathcal{F}} \ln \left|\left(f_{1} \circ \cdots \circ f_{n}\right)^{\prime}(x)\right|
$$

называется показателем Ляпунова растяжения в точке $x$.

Хёрдер [10] показал, что для минимального действия конечно порожденной группы $G \subset \operatorname{Diff}^{2}\left(S^{1}\right)$ функция $\lambda_{\exp }(\cdot, \mathcal{F})$ почти всюду по мере Лебега совпадает с некоторой константой $\lambda_{\exp }(G, \mathcal{F})$, называемой показателем Ляпунова растяжения группь $G$. Хотя величина этого показателя зависит от выбора системы образующих, его равенство нулю или положительность от этого выбора не зависит. В предположении положительности показателя растяжения теорема Хёрдера [10] утверждает, что действие группы эргодично относительно меры Лебега.

Однако оказывается, что экспоненциальное растяжение для N-группы невозможно не только для «жадного» алгоритма:

Теорема 4. Показатель Ляпунова растяжения любой $N$-группъ $G$ равен нулю, $\lambda_{\exp }(G)=0$.

В частности, эргодичность действия такой группы не может быть выведена из теоремы Хёрдера.

Напротив, для групп с нулевым показателем растяжения стационарные меры для конечно порожденной случайной динамики сингулярны (см. [4, следствие 1.22]). Таким образом, имеет место 
Следствие 2. Пусть $G$ есть $N$-группа, a $m$ - вероятностная мера на ней, носитель которой состоит из конечного числа элементов и порождает $G$ как полугруппу. Тогда (единственная) $m$-стационарная мера сингулярна относительно меры Лебега.

Для действия группы $P S L(2, \mathbb{Z})$ аналогичное утверждение было анонсировано Гиваршем и Ле Жаном [7] и доказано в [8, предложение 15].

В заключение сделаем несколько замечаний о возможных обобщениях:

Замечание 2. Все доказательства в настоящей статье используют только «локальную» технику. Поэтому все результаты остаются верными и при замене групп на псевдогруппы, в частности, допуская формулировку для слоений коразмерности 1 .

Замечание 3. Результаты данной статьи допускают также обобщение на случай действий с канторовым минимальным множеством. В этом случае свойство $(\star)$ из работы [4] заменяется на введенное в той же работе свойство $(\Lambda \star)$ : требования из определения 2 накладываются лишь на нерастяжимые точки из минимального множества. При этом при построении марковского разбиения используются не только собственно нерастяжимые точки и их орбиты, но и интервалы - компоненты связности дополнения до канторова множества (для которых аналогом энергии служит величина $1 /|J|)$. Таким образом, получающиеся интервалы марковского разбиения могут быть ограничены не только точками, принадлежащими орбитам нерастяжимых точек, но и концевыми точками интервалов дополнения.

1.3. Структура статьи. В $\S 2$ мы докажем теорему 1 , построив процедуру растяжения и описав ее свойства. После этого везде далее в статье мы будем считать указанную в заключении теоремы 1 систему интервалов и растягивающих отображений выбранной и зафиксированной. Затем в 33 мы напомним необходимые для дальнейших рассуждений сведения из [4] и докажем несколько вспомогательных утверждений.

$\S 4$ посвящен доказательству теоремы 2. В разд. 4.1 мы выведем теорему 2 из нескольких технических лемм, а в разд. 4.2 эти леммы докажем. Наконец, в §5 мы докажем следствие 1.

Как уже было сказано выше, доказательства теорем 3 и 4 публикуются в отдельной работе.

Благодарности. Авторы хотели бы поблагодарить Э. Жиса, Ю. С. Ильяшенко, Д. В. Аносова, Б. Деруана, А. Наваса, М. Ю. Любича, И. Гиварша и И. Г. Хованскую за ценные обсуждения и интерес к работе. Кроме того, авторы глубоко признательны рецензенту за огромную проделанную им работу.

\section{§2. Доказательство теоремы 1}

Напомним сначала конструкцию процедуры растяжения, приведенную в [4], удовлетворяющую условиям (ii)-(iv) теоремы 1. А именно, выберем для каждой нерастяжимой точки $x^{*} \in \mathrm{NE}$ отображения $g_{+}$и $g_{-}$, для которых она является (топологически) отталкивающей соответственно справа и слева.

Для каждой точки из правой (левой) окрестности точки $x^{*}$ мы можем рассмотреть первую итерацию отображения $g_{+}\left(\right.$соответственно $\left.g_{-}\right)$, «выбрасывающую» эту точку за пределы некоторой (фиксированной) окрестности точки $x^{*}$. Как и предполагает формулировка теоремы, оказывается, что при подходящем 
выборе окрестностей такая «итерация выхода» является растягивающей с равномерной по начальной точке оценкой снизу на производную.

Более точно, выберем правую окрестность $I^{+}=\left[x^{*}, x^{*}+\delta\right]$ точки $x^{*}$, целиком содержащуюся в правом бассейне $g_{+}$-отталкивания этой точки. Потребуем при этом, чтобы $\delta$ было достаточно мало, чтобы образ $g_{+}\left(x^{*}+\delta\right)$ был по меньшей мере вдвое ближе к $x^{*}$, чем любая отличная от $x^{*}$ нерастяжимая точка, и, кроме того (нам это потребуется в дальнейшем), чтобы правая граница $x^{*}+\delta$ принадлежала (всюду плотному) множеству $G(\mathrm{NE})$.

Разобьем $I^{+}$на $g_{+}$-итерации фундаментальной области $J^{+}:=g_{+}\left(I^{+}\right) \backslash I^{+}$:

$$
I^{+}=\bigcup_{k=1}^{\infty} g_{+}^{-k}\left(J^{+}\right) \text {. }
$$

Тогда, как следует из [4, лемма 5.7] (мы воспроизведем формулировку этой леммы ниже, см. лемму 1), при всех $k$, больших некоторого $k_{0}$, выполнено условие

$$
\left(g_{+}^{k}\right)^{\prime}(x) \geqslant 2 \text { для любых } x \in g_{+}^{-k}\left(J^{+}\right) .
$$

Положим $\widetilde{I}^{+}:=g_{+}^{-k_{0}}\left(I^{+}\right), \widetilde{J}^{+}:=g_{+}^{-k_{0}}\left(J^{+}\right)$и сопоставим интервалу $\widetilde{I}^{+}$отображение $g_{+}$, а интервалу $\widetilde{J}^{+}$отображение $g_{+}^{k_{0}}$.

Взяв так построенные интервалы $\widetilde{I}^{+}$и $\widetilde{J}^{+}$для всех нерастяжимых точек и аналогичные интервалы в левых окрестностях, мы получаем набор интервалов и соответствующих им отображений, для которых выполнены заключения (iii) и (iv) теоремы 1.

Теперь дополнение к объединению $U$ таких интервалов - компакт, отделенный от множества нерастяжимых точек; поэтому

для любого $x \in S^{1} \backslash U$ существует $g \in G$, такой, что $g^{\prime}(x)>1$.

Неравенство $g^{\prime}(x)>1$ выполнено автоматически и в некоторой окрестности точки $x$, которую можно выбрать так, чтобы производная отображения $g$ в ней была отделена от единицы. Таким образом, мы имеем открытое покрытие множества $S^{1} \backslash U$, из которого в силу компактности можно выделить конечное подпокрытие. Следовательно, $S^{1} \backslash U$ можно разбить на интервалы $I_{i}$, каждому из которых соответствует отображение $g_{i} \mathrm{c} \inf _{x \in I_{i}} g_{i}^{\prime}(x)>1$. Осталось положить $\lambda_{0}:=\min _{i} \inf _{x \in I_{i}} g_{i}^{\prime}(x)$ и заметить, что заключение (ii) выполнено.

Наконец, отметим, что поскольку множество $G(\mathrm{NE})$ всюду плотно, можно выбрать интервалы $I_{i}$ с концами из этого множества (опять-таки мы воспользуемся этим позднее). Мы получаем разбиение окружности, удовлетворяющее заключениям (ii)-(iv) теоремы, хотя, быть может, не удовлетворяющее условию (i) - марковости. Мы будем называть его предварителъным разбиением.

Заметим теперь, что каждая из граничных точек построенных интервалов за конечное число итераций процедуры растяжения переходит в нерастяжимую. Действительно, с одной стороны, для нерастяжимой точки $x^{*}$ в ее образе $g\left(x^{*}\right)$ под действием некоторого отображения $g \in G$ производная любого отображения $\tilde{g} \in G$ не может превосходить $1 / g^{\prime}\left(x^{*}\right)$ - в противном случае композиция $\tilde{g} \circ g$ будет растягивать в $x^{*}$. С другой стороны, при итерировании процедуры растяжения ни одна точка, кроме нерастяжимых, не может бесконечно оставаться в прилегающих к нерастяжимым точкам интервалах. В силу условий (ii)-(iv), если в процессе растяжения точка провела $n$ итераций вне этих интервалов, то производная соответствующей композиции в этой точке не меньше $\lambda^{n}$. 
Поэтому процедура растяжения для граничных точек (как принадлежащих орбитам нерастяжимых) не может продолжаться бесконечно и, значит, заканчивается попаданием каждой такой точки в какую-либо из нерастяжимых.

Подразобьем теперь предварительное разбиение, добавив к его концам все их образы при итерациях (предварительной) процедуры растяжения. При этом, если подразбиваемый интервал не примыкает к нерастяжимой точке, мы оставляем отображение, соответствующее его частям, тем же, что и для исходного интервала.

Наконец, каждый интервал $\widetilde{I}^{+}$предварительного разбиения, примыкающий (для определенности, справа) к одной из нерастяжимых точек $x^{*}$, мы дополнительно подразобьем $g_{+}$-прообразами $\left\{g_{+}^{-j}\left(x^{*}+\delta\right)\right\}_{j=k_{0}+1}^{n}$ его правой точки $g_{+}^{-k_{0}}\left(x^{*}+\delta\right)$, выбрав $n$ достаточно большим, чтобы ближайшая к $x^{*}$ точка была бы таким прообразом. После этого (см. рис. 1) оставим на примыкающей к $x^{*}$ части $\hat{I}$ интервала $\widetilde{I}^{+}$отображение $g_{+}$, а каждому из оставшихся подынтервалов $I \subset \widetilde{I}^{+}$сопоставим отображение $g_{+}^{k_{+}(I)}$, где $k_{+}(I)$ определено условием $g_{+}^{k_{+}(I)} \subset J^{+}$. Аналогично поступим со всеми примыкающими к нерастяжимым точкам слева интервалам. Легко видеть, что при этом продолжает выполняться условие (iv), поскольку при итерации, следующей за выходом из нового интервала, точки оказываются там же, где и при итерации, следующей за выходом из интервала $\widetilde{I}^{+}$при предварительной процедуре.

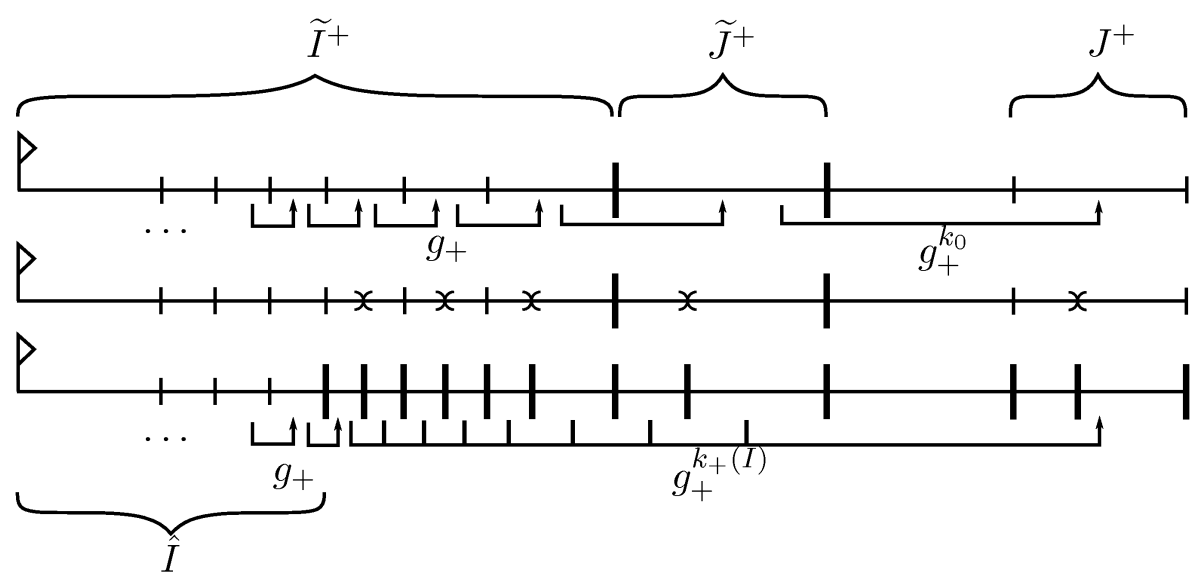

Рис. 1. Процедура подразбиения

С другой стороны, добавив образы граничных точек предварительного разбиения, мы обеспечили марковское свойство (i). Действительно, на каждом из интервалов нового разбиения соответствующее ему отображение является композицией соответствующих отображений для предварительного разбиения; поэтому образы граничных точек нового разбиения также ему принадлежат, что и означает выполнение условия (i).

Итак, новое разбиение удовлетворяет всем условиям (i)-(iv), и теорема 1 доказана. 


\section{§3. Вспомогательные сведения}

В этом параграфе мы напомним основные понятия и факты, касающиеся контроля искажения. Большинство из них являются классическими для одномерной динамики (эти идеи восходят к работам Данжуа, Салливана, Катка, Эрмана), так что мы ограничимся лишь упоминанием нужных нам формулировок и определений. Более детальный обзор и доказательства можно найти, например, в [12], [4], [5], [16]. Наконец, как и было указано во введении, мы будем считать, что система отображений из теоремы 1 выбрана и зафиксирована.

Определение 5. Определим коэфбициент искажения на $I C^{2}$-гладкого диффеоморфизма $F: I \rightarrow J$ формулой

$$
\varkappa(F ; I):=\log \left(\frac{\max _{I} F^{\prime}}{\min _{I} F^{\prime}}\right),
$$

а соответствующую норму искажения - формулой

$$
\eta(F ; I):=\sup _{I^{\prime} \subset I} \frac{\varkappa\left(F ; I^{\prime}\right)}{\left|F\left(I^{\prime}\right)\right|}=\max _{J}\left|\left(\log \left(\left(F^{-1}\right)^{\prime}\right)\right)^{\prime}\right| .
$$

Второе равенство здесь несложно выводится из теоремы Лагранжа; также из теоремы Лагранжа легко получить, что для коэффициента искажения имеет место оценка $\varkappa(F, I) \leqslant C_{(F)}|I|$, где константа $C_{(F)}$ зависит лишь от отображения $F \in \operatorname{Diff}^{2}\left(S^{1}\right)$.

Легко проверить, что коэффициент искажения субаддитивен относительно операции композиции. Для нормы вместо условия субаддитивности выполняется похожее условие:

$$
\eta(f \circ g ; I) \leqslant \eta(f ; g(I))+\frac{\eta(g ; I)}{\min _{x \in g(I)}\left|f^{\prime}(x)\right|} .
$$

Непосредственным следствием оценки на коэффициент искажения является

Предложение 1. Пусть $\mathcal{F}-$ конечное подмножество в $\operatorname{Diff}_{+}^{2}\left(S^{1}\right) u f_{1}, \ldots$, $f_{n}$ - отображения из $\mathcal{F}$. Тогда для любого интервала I на окружности выполняется следующая оценка:

$$
\varkappa\left(f_{n} \circ \cdots \circ f_{1} ; I\right) \leqslant C_{\mathcal{F}} \sum_{i=0}^{n-1}\left|f_{i} \circ \cdots \circ f_{1}(I)\right|,
$$

где константа $C_{\mathcal{F}}$ зависит только от выбранного множества $\mathcal{F}$.

Другими словами, оценка суммы длин промежуточных образов исходного интервала при вычислении композиции отображений дает оценку коэффициента искажения этой композиции. Так, например, если для композиционной степени некоторого отображения сумма длин промежуточных образов равномерно (по степени) ограничена, то и коэффициент искажения оказывается равномерно ограниченным.

Прямым применением этой техники является следующая

Лемма 1 [4, лемма 5.7]. Пусть $x_{0}$ - неподвижная точка $C^{2}$-гладкого диффеоморфизма $f:\left[x_{0}, a\right] \rightarrow\left[x_{0}, b\right]$, причем $f(x)>x$ для всех $x \in\left(x_{0}, a\right]$. Пусть 
$F:\left[x_{0}, a\right] \rightarrow[a, b]$ - отображсние первого попадания в интервал $J=[a, b], a$ именно

$$
F(x):=f^{k(x)}(x), \quad k(x):=\min \left\{k \geqslant 1 \mid f^{k}(x) \in J\right\} .
$$

Рассмотрим бесконечное множество интервалов $J_{k}:=f^{-k}(J)$, на каждом из которых отображсение $F$ непрерьвно и совпадает $c f^{k}$ всюду за исключением правой граничной точки, и положим $f_{k}:=\left.f^{k}\right|_{J_{k}}: J_{k} \rightarrow J$. Тогда

(i) нормы искажения отображсений $f_{k}$ равномерно ограничены;

(ii) начиная с некоторого $k_{0}$, отображения $f_{k}$ равномерно растягивают; более точно, найдется такое $\lambda_{1}>1$, что для всех $k \geqslant k_{0}$ u всех $x \in J_{k}$ имеет место неравенство $f_{k}^{\prime}(x) \geqslant \lambda_{1}$. Более того, выбирая $k_{0}$ достаточно большим, можно добиться выполнения этого условия с $\lambda_{1}=2$.

В дальнейшем нам потребуется следующее семейство разбиений окружности, порождаемое итерациями процедуры растяжения:

Определение 6. Обозначим через $\Delta_{0}$ множество концов интервалов марковского разбиения $\mathcal{I}$. Определим по индукции множество концов $\Delta_{k}(I)$ интервалов разбиения $k$-го уровня на интервале $I \in \mathcal{I}$, после чего положим $\Delta_{k}=$ $\bigcup_{I \in \mathcal{I}} \Delta_{k}(I)$.

Для интервала $I$, не прилегающего к нерастяжимым точкам, положим

$$
\Delta_{k+1}(I)=g_{I}^{-1}\left(\Delta_{k} \cap g_{I}(I)\right) .
$$

Для интервала $I$, у которого один из концов - нерастяжимая точка, положим

$$
\Delta_{k+1}(I)=\bigcup_{j=1}^{\infty} g_{I}^{-j}\left(\Delta_{k} \cap\left(g_{I}(I) \backslash I\right)\right) .
$$

Множество интервалов, на которые окружность разбивается точками из $\Delta_{k}$, будем называть разбиением $k$-го уровня и обозначать через $\mathcal{I}_{k}$.

Определение 7. Для интервала $I \subset S^{1}$ определим отображение $F_{I}$ как первое в последовательности отображений растяжения $G_{n, I}$, для которого

- либо образ $G_{n, I}(I)$ пересекает множество $\Delta_{0}$ граничных точек марковского разбиения по своей внутренней точке;

- либо $G_{n, I}(I)$ содержится в интервале $I^{\prime} \in \mathcal{I}$ марковского разбиения, прилегающем к некоторой нерастяжимой точке, и при этом пересекает $\Delta_{1}\left(I^{\prime}\right)$ по своей внутренней точке.

Кроме того, в дополнение к максимальному в этой же последовательности растяжений отображению $G_{I}$ мы рассмотрим и предыдущее перед ним: если $G_{I}=$ $G_{m, I}$, то положим $\widetilde{G}_{I}:=G_{m-1, I}$. Это определение удобно тем, что для любого интервала $I \in \mathcal{I}_{k}$ разбиения любого уровня $k \geqslant 1$ образ $\widetilde{G}_{I}(I)$ в точности марковский: $\widetilde{G}_{I}(I) \in \mathcal{I}$.

Предложение 2. Для интервала $I \subset S^{1}$ норма искажения его растяжения $F_{I}$ допускает равномерную оценку: найдется такая константа $C_{\eta}$, что

$$
\eta\left(F_{I} ; I\right) \leqslant C_{\eta} \text { для любого } I \subset S^{1} .
$$

Доказательство. Рассмотрим последовательность растяжения $I_{(j)}=G_{j, I}(I)$, $j=1, \ldots, m$, интервала $I$, завершающуюся интервалом $F_{I}(I)=G_{m, I}(I)$. Отметим в ней подпоследовательность $I_{\left(j_{k}\right)}, k=1, \ldots, n$, тех интервалов, предшествующие которым не содержатся в прилегающих к нерастяжимым точкам 
интервалах разбиения. Легко видеть, что $I_{\left(j_{n}\right)}=F_{I}(I)$ : если бы интервал $I_{\left(j_{n}-1\right)}$ содержался в прилегающем к нерастяжимой точке интервале $I^{\prime}$, то он бы пересекал $\Delta_{1}\left(I^{\prime}\right)$ и процедура растяжения была бы остановлена раньше.

Из свойств процедуры растяжения (см. замечание 1) следует, что

$$
\left|I_{\left(j_{k+1}\right)}\right| /\left|I_{\left(j_{k}\right)}\right| \geqslant \min \left(\lambda_{0}, 2\right)=\lambda>1 .
$$

Покажем теперь, что сумма длин $\sum_{i=j_{k-1}+1}^{i=j_{k}}\left|I_{(i)}\right|$ допускает оценку сверху $C_{F}\left|I_{\left(j_{k}\right)}\right|$, где $C_{F}$ - некоторая (не зависящая от исходного интервала) константа. Действительно, обозначим через $g_{+}$итерируемое на этом участке отображение, через $J$ соответствующую фундаментальную область и через $J_{l}=g_{+}^{-l}(J)$ ее прообразы. Тогда $\left|I_{\left(j_{k}-1\right)}\right| \leqslant$ const $\cdot\left|I_{\left(j_{k}\right)}\right|$, поскольку эти два интервала отличаются на применение одного из отображений $g_{j}$. Далее, если $j_{k-1}+1=j_{k}$, то доказывать нечего: $\sum_{i=j_{k-1}+1}^{i=j_{k}}\left|I_{(i)}\right|=\left|I_{\left(j_{k}\right)}\right|$. В противном случае в силу определения последовательности $j_{k}$ все интервалы $I_{(i)}$ содержатся в одном из прилегающих к некоторой нерастяжимой точке интервалов $I_{j}^{ \pm}$. Мы обозначим этот интервал через $I^{+}$, а соответствующее ему отображение через $g_{+}$. Тогда на отрезке итераций $i=j_{k-1}+1, \ldots, j_{k}-1$ итерируется отображение $g_{+}$, и по определению последовательности $j_{k}$ в заключительный момент имеем $I_{\left(j_{k}-1\right)} \subset g_{+}\left(I^{+}\right) \backslash I^{+}$.

Обозначив соответствующую фундаментальную область через $J:=g_{+}\left(I^{+}\right) \backslash I^{+}$ и положив $J_{l}:=g_{+}^{-l}(J)$, получим $I_{\left(j_{k}-l\right)} \subset J_{l-1}\left(\right.$ при $\left.l \leqslant j_{k}-j_{k-1}-1\right)$. Таким образом, длина интервалов $I_{\left(j_{k}-l\right)}$ допускает оценку

$$
\left|I_{\left(j_{k}-l\right)}\right|=\left|g_{+}^{-l+1}\left(I_{\left(j_{k}-1\right)}\right)\right| \leqslant e^{\varkappa\left(g^{-l+1} ; J\right)} \frac{\left|J_{l-1}\right|}{|J|}\left|I_{\left(j_{k}-1\right)}\right| .
$$

Отсюда в силу леммы 1

$$
\sum_{l}\left|I_{\left(j_{k}-l\right)}\right| \leqslant \text { const } \cdot \frac{\sum_{l}\left|J_{l-1}\right|}{|J|}\left|I_{\left(j_{k}-1\right)}\right| \leqslant \text { const } \cdot\left|I_{\left(j_{k}-1\right)}\right| \leqslant \text { const } \cdot\left|I_{\left(j_{k}\right)}\right| .
$$

Выражение (2) и доставляет требуемую оценку: мы выберем $C_{F}$ как константу в его правой части.

Из этой оценки получаем

$$
\begin{aligned}
\sum_{i=1}^{m}\left|I_{(i)}\right| & =\sum_{k=1}^{n} \sum_{i=j_{k-1}+1}^{j_{k}}\left|I_{(i)}\right| \leqslant \sum_{k=1}^{n} C_{F}\left|I_{\left(j_{k}\right)}\right| \\
& \leqslant C_{F}\left(\left|I_{\left(j_{n}\right)}\right|+\frac{\left|I_{\left(j_{n}\right)}\right|}{\lambda}+\frac{\left|I_{\left(j_{n}\right)}\right|}{\lambda^{2}}+\cdots\right) \leqslant \frac{C_{F}}{1-1 / \lambda}\left|I_{\left(j_{n}\right)}\right|=C\left|F_{I}(I)\right| .
\end{aligned}
$$

Более того, точно так же сумма длин соответствующих прообразов любого подынтервала $I^{\prime} \subset F_{I}(I)$ оценивается числом $C\left|I^{\prime}\right|$. Применяя предложение $1 \mathrm{k}$ набору $\mathcal{F}=\left\{g_{I} \mid I \in \mathcal{I}\right\}$ соответствующих разбиению отображений и используя определение нормы искажения, получаем оценку

$$
\eta\left(F_{I} ; I\right) \leqslant C \cdot C_{\mathcal{F}}=: C_{\eta}
$$

Одним из ключевых элементов дальнейших рассуждений будет следующее 
Определение 8. Энергией точки $x \in S^{1}$ назовем конечный или бесконечный супремум

$$
E(x):=\sup \left\{g^{\prime}(x) \mid g \in G\right\} .
$$

Кроме того, мы будем называть энергетической областью множество $\mathcal{E}(C):=$ $\{x \mid E(x)<C\}$.

Замечание 4. Легко видеть, что

$$
E(g(x))=E(x) / g^{\prime}(x) .
$$

Лемма $2[4$, теорема A]. $E(x)<\infty$ тогда и только тогда, когда $x \in G(\mathrm{NE})$. В дальнейшем нам потребуется следующая

Лемма 3. Для любого $C$ энергетическая область $\mathcal{E}(C)$ состоит из конечного числа точек.

Доказательство. Для произвольной нерастяжимой точки $x^{*}$, прилегающего к ней справа интервала $I^{+}$разбиения и соответствующего ему отображения $g^{+}$рассмотрим разбиение интервала $I^{+}$на образы фундаментальной области,

$$
I^{+}=\bigcup_{k=1}^{\infty} g_{+}^{-k}\left(J^{+}\right), \quad J^{+}:=g_{+}\left(I^{+}\right) \backslash I^{+} .
$$

Тогда длины $\left|g_{+}^{-k}\left(J^{+}\right)\right|$стремятся к нулю; поэтому из оценки на норму искажения из леммы 1 следует, что для некоторого $k_{1}$ при всех $k \geqslant k_{1}$ имеет место неравенство $\left(g_{+}^{k}\right)^{\prime}(x)>C$ для всех $x \in g_{+}^{-k}\left(J^{+}\right)$. Следовательно, точка с энергией, меньшей $C$, не может попадать при применении к ней процедуры растяжения в правую окрестность $g_{+}^{-k_{1}}\left(I^{+}\right)$.

Взяв максимум таких чисел $k_{1}$ по всем (правым и левым) окрестностям точек $x^{*} \in \mathrm{NE}$, обозначим его через $k^{\prime}$. Тогда при итерациях процедуры растяжения для точки с энергией, меньшей $C$, не применяется подряд более $k^{\prime}-1$ соответствующих интервалам $I_{i}^{ \pm}$отображений $g_{i}^{ \pm}$. Кроме того, производная растягивающей композиции, в которой $n$ раз встречаются отображения $g_{j}$, не меньше $\lambda^{n}$. Поэтому процедура растяжения переводит точку с энергией, меньшей $C$, в нерастяжимую за не больше чем $k^{\prime}\left\lceil\log _{\lambda} C\right\rceil$ итераций.

Но точка однозначно восстанавливается по своему образу и последовательности применяемых отображений; поэтому таких точек не больше чем

$$
\# \mathrm{NE} \cdot\left(s^{k^{\prime}\left\lceil\log _{\lambda} C\right\rceil}+s^{k^{\prime}\left\lceil\log _{\lambda} C\right\rceil-1}+\cdots+1\right)
$$

где $s$ - число различных отображений среди $g_{I}, I \in \mathcal{I}$.

В следующем параграфе, как и при доказательстве теоремы 1, нам неоднократно потребуется работать в правой (либо левой) окрестности одной из нерастяжимых точек. В этом случае мы будем обозначать через $x^{*}$ саму нерастяжимую точку, через $I^{+}$прилегающий к ней справа интервал разбиения, а через $g_{+}=g_{I^{+}}$соответствующее отображение. Кроме того, мы будем обозначать соответствующую фундаментальную область через $J:=g_{+}\left(I^{+}\right) \backslash I^{+}$, a eе прообразы под действием $g_{+}$через $J_{k}:=g_{+}^{-k}(J)$.

Нам при этом также будет полезно следующее замечание, легко следующее из того, что $g_{+}^{\prime}\left(x^{*}\right)=1$ :

Замечание 5. Отношение длин соседних образов фундаментальной области стремится к единице: $\left|J_{k+1}\right| /\left|J_{k}\right| \rightarrow 1$ при $k \rightarrow \infty$. 


\section{§4. Доказательство теоремы 2}

\section{1. Формулировки вспомогательных утверждений и доказатель-} ство теоремы 2. Основными инструментами при доказательстве теоремы 2 будут процедура растяжения и функция энергии. А именно, соотношение (3) можно рассматривать как соотношение на диффеоморфизм $g \in G$ при заданной функции энергии $E$.

Это соотношение оказывается достаточно «жестким»: так, предложение 3 ниже утверждает, что среди сохраняющих данный марковский интервал диффеоморфизмов, являющихся ограничениями элементов группы $G$, тождественный оказывается $C^{1}$-изолированной точкой. Поэтому (см. лемму 4) отображений из заданного марковского интервала в заданный с известной оценкой на искажение может быть лишь конечное число.

Далее, «увеличение» с помощью процедуры растяжения позволяет получать отображения с оценкой на норму искажения, а контроль энергии при этом ограничивает множество возможных интервалов (см. лемму 6).

Теперь сформулируем необходимые для доказательства теоремы 2 утверждения.

Предложение 3 (о тождественности). Существует $\varepsilon^{\prime}>0$, такое, что если для некоторого $k_{0}$ интервал $I$ принадлежит $\mathcal{I}_{k_{0}}$, а для отображения $g \in G$ имеет место равенство $g(I)=I u \operatorname{dist}_{C^{1}}\left(\left.g\right|_{I}\right.$, id $)<\varepsilon^{\prime}$, mo $\left.g\right|_{I}=\mathrm{id}$.

Лемма 4 (о конечности). Пусть $I$-марковский интервал, $I^{\prime} \subset S^{1}$. Тогда для любого $C>0$ есть лишь конечное число дифбеоморфизмов $g: I \rightarrow I^{\prime}$, являющихся ограничениями отображений из $G$, для которых $\eta(g, I) \leqslant C$.

Лемма 5 (о стабилизаторе). Пусть $x^{*} \in \mathrm{NE}$. Тогда существует такой конечный набор отображений $h_{1}, \ldots, h_{s} \in G, h_{j}\left(x^{*}\right)=x^{*}$, что любое отображсение $g \in G$ с $g\left(x^{*}\right)=x^{*}$ в некоторой правой окрестности $U=\left[x^{*}, x^{*}+\delta\right)$ представимо в виде $\left.g\right|_{U}=h_{j} \circ g_{+}^{k}$ для некоторьх $j \in\{1, \ldots, s\}, k \in \mathbb{Z}$. Иными словами, $\left\langle g_{+}\right\rangle$ является подгруппой конечного индекса в стабилизаторе $\operatorname{Stab}\left(x^{*}\right)$, paсcматриваемом как подгруппа группы правых ростков. Кроме того, отображсения $h_{j}$ коммутируют с $g_{+}$в достаточно малой правой окрестности точки $x^{*}$.

Замечание 6. Заменяя каждый элемент $h$ на его композицию с достаточно большой отрицательной степенью отображения $g_{+}$, можно добиться, чтобы отображения $h$ и $g_{+}$коммутировали на всем прилегающем к точке $x^{*}$ интервале $I^{+} \in \mathcal{I}$. Действительно, если равенство $h \circ g_{+}=g_{+} \circ h$ выполнено в некоторой правой окрестности $U^{+}$, то равенство $\left(h \circ g_{+}^{-l}\right) \circ g_{+}=g_{+} \circ\left(h \circ g_{+}^{-l}\right)$ выполнено уже на $g_{+}^{l}\left(U^{+}\right)$. В дальнейшем мы будем считать, что все отображения $h_{i}$ из заключения леммы 5 выбраны коммутирующими с $g_{+}$на всем $I^{+}$.

Лемма 6 (об образе растяжения). Для любой константы $C>0$ существуют $C^{\prime}, E_{0}, \varepsilon_{0}$, обладающие следующим свойством. Если для некоторого интервала $J \subset S^{1}$ существует $g \in G$, переводящее $J$ в марковский интервал $I=g(J) \in \mathcal{I}$ с $\eta(g, J) \leqslant C$, то на некотором шаге $n$ процедуры растяэения

(i) $\eta\left(G_{n, J}, J\right) \leqslant C^{\prime}$,

(ii) энергия концов интервала $G_{n, J}(J)$ не превосходит $E_{0}$,

(iii) $\left|G_{n, J}(J)\right| \geqslant \varepsilon_{0}$.

Доказательство теоремы 2. Предположим сначала, что каждая нерастяжимая точка является единственной нерастяжимой точкой на своей орбите. 
Рассмотрим (заданное определением 6) разбиение первого уровня $\mathcal{I}_{1}$. Его отрезки накапливаются к нерастяжимым точкам и только к ним. Поэтому сначала мы научимся описывать поведение $g$ в правой и левой окрестностях каждой из этих точек.

Пусть точка $x^{*}$ нерастяжимая. Тогда $g\left(x^{*}\right) \in G(\mathrm{NE})$, и за конечное число $n_{0}$ итераций последовательность растяжений правой окрестности точки $g\left(x^{*}\right)$ переведет саму точку $g\left(x^{*}\right)$ в нерастяжимую (см. рассуждения в доказательстве теоремы 1 при проведении подразбиения). В силу сделанного предположения $G_{g\left(x^{*}\right), n_{0}}^{+}\left(g\left(x^{*}\right)\right)=x^{*}$.

Пусть прилегающий к $x^{*}$ справа интервал - это $I^{+}=\left[x^{*}, y\right]$; положим $y_{k}=g_{+}^{-k}(y)$. Отметим, что $G_{g\left(x^{*}\right), n_{0}}^{+} \circ g \in \mathrm{Stab}^{+}\left(x^{*}\right)$. Поэтому по лемме 5 в достаточно малой правой окрестности точки $x^{*}$ эта композиция имеет вид $h_{i} \circ g_{+}^{l}$, где $l \in \mathbb{Z}, h_{i}$ - одно из конечного набора отображений. Таким образом, при всех достаточно больших $k$ на интервале $\left[x^{*}, y_{k}\right]$ выполнены равенства

$$
\left.G_{g\left(x^{*}\right), n_{0}+k-l}^{+} \circ g\right|_{\left[x^{*}, y_{k}\right]}=\left.g_{+}^{k-l} \circ\left(G_{g\left(x^{*}\right), n_{0}}^{+} \circ g\right)\right|_{\left[x^{*}, y_{k}\right]}=\left.g_{+}^{k-l} \circ\left(h_{i} \circ g_{+}^{l}\right)\right|_{\left[x^{*}, y_{k}\right]} \text {. }
$$

Поскольку отображения $h_{i}$ были выбраны (см. замечание 6) коммутирующими с $g_{+}$на $I^{+}$, композиция равенств $(4)$ с $\left(G_{x^{*}, k}^{+}\right)^{-1}=g_{+}^{-k}: I^{+} \rightarrow\left[x^{*}, y_{k}\right]$ дает

$\left.G_{g\left(x^{*}\right), n_{0}+k-l}^{+} \circ g \circ\left(G_{x^{*}, k}^{+}\right)^{-1}\right|_{I^{+}}=\left.g_{+}^{k-l} \circ\left(h_{i} \circ g_{+}^{l}\right) \circ g_{+}^{-k}\right|_{I^{+}}=\left.g_{+}^{k-l} \circ h_{i} \circ g_{+}^{-(k-l)}\right|_{I^{+}}=h_{i}$.

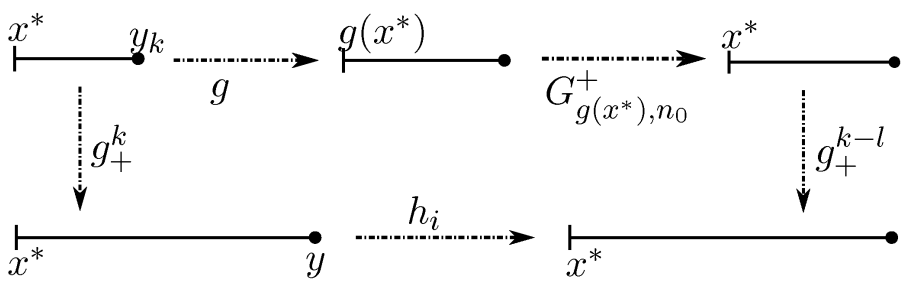

Рис. 2. Коммутативная диаграмма

Таким образом, при всех достаточно больших $k$

$$
\left.g\right|_{\left[x^{*}, y_{k}\right]}=\left(G_{g\left(x^{*}\right), n_{0}+k-l}^{+}\right)^{-1} \circ h_{i} \circ G_{x^{*}, k}^{+} .
$$

Аналогично рассматриваются левые окрестности нерастяжимых точек.

Дополнение к этим окрестностям является объединением конечного числа интервалов из $\mathcal{I}_{1}$. Перейдем в этих интервалах к разбиению $\mathcal{I}_{2}$ второго уровня. В нем вновь накопление интервалов происходит только к точкам, которые за одну итерацию растяжения переходят в нерастяжимые. Так же, как и ранее, для каждой из этих точек накопления существует окрестность, в которой имеет место представление (5). Дополнение до этих окрестностей опять содержит конечное число интервалов второго уровня.

Продолжая, мы разбиваем всю окружность на окрестности прообразов нерастяжимых точек, в которых имеет место представление (5), и конечное число интервалов разбиения $\mathcal{I}_{k} k$-го уровня для произвольно большого $k$. Как только диаметр такого разбиения становится достаточно малым, оказываются применимыми леммы 4 и 6. 
А именно, пусть $I-$ интервал одного из разбиений $\mathcal{I}_{k}$. Тогда (см. определение 7) предпоследним в его последовательности растяжения $\left\{G_{i, I}(I)\right\}$ идет некоторый марковский интервал $\tilde{I}=\widetilde{G}_{I}(I)=G_{n, I}(I) \in \mathcal{I}$. Заметим теперь, что норма искажения отображения $\widetilde{G}_{I}$ на $I$ допускает равномерную оценку. Действительно, норма искажения на $I$ отображения $F_{I}=G_{I}$ не превосходит $C_{\eta}$ в силу предложения 2 ; с другой стороны, $G_{I}=g_{\tilde{I}}$ ○ $\widetilde{G}_{I}$. Отсюда следует, что

$$
\eta\left(\widetilde{G}_{I} ; I\right)=\eta\left(g_{\tilde{I}}^{-1} \circ F_{I}, I\right) \leqslant \eta\left(g_{\tilde{I}}^{-1}, g_{\tilde{I}}(\tilde{I})\right)+\frac{1}{\min _{\tilde{I}}\left(g_{\tilde{I}}^{-1}\right)^{\prime}} C_{\eta} .
$$

Остается заметить, что как первое слагаемое в правой части неравенства (6), так и коэффициент перед $C_{\eta}$ принимают лишь конечное множество значений (поскольку интервал $\tilde{I}$ марковский), и потому правая часть в (6) не превосходит некоторой константы $C_{\eta}^{\prime}$.

Тогда для нормы искажения отображения $\widetilde{G}_{I} \circ g^{-1}$ на $g(I)$ имеем

$$
\begin{aligned}
\eta\left(\widetilde{G}_{I} \circ g^{-1} ; g(I)\right) & \leqslant \eta\left(\widetilde{G}_{I} ; I\right)+\frac{\eta\left(g^{-1} ; g(I)\right)}{\min _{I} \widetilde{G}_{I}^{\prime}} \leqslant \eta\left(\widetilde{G}_{I} ; I\right)+e^{\varkappa\left(\widetilde{G}_{I} ; I\right)} \frac{|I|}{|\tilde{I}|} \eta\left(g^{-1} ; g(I)\right) \\
& \leqslant C_{\eta}^{\prime}+\text { const } \cdot|I| \eta\left(g^{-1}, g(I)\right) .
\end{aligned}
$$

Третий сомножитель в правой части (7) равномерно ограничен сверху; поэтому мы можем предъявить $\varepsilon_{1}>0$, такое, что при $|I|<\varepsilon_{1}$

$$
\eta\left(\widetilde{G}_{I} \circ g^{-1} ; g(I)\right) \leqslant 2 C_{\eta}^{\prime} .
$$

Применим теперь лемму 6 к интервалу $J=g(I)$ с константой $C=2 C_{\eta}^{\prime}$ : мы видим, что для некоторых констант $C^{\prime}, E_{0}, \varepsilon_{0}$ в последовательности растяжения интервала $J=g(I)$ найдется интервал $I^{\prime}=G_{m, J}(J)$ длины не меньше $\varepsilon_{0}$, энергия концов которого не превосходит $E_{0}$, с $\eta\left(G_{m, J}, J\right) \leqslant C^{\prime}$.

Далее, в силу леммы 3 множество $\mathcal{E}\left(E_{0}\right)$ конечно, и потому интервал $I^{\prime}$ принадлежит конечному множеству интервалов с концами в $\mathcal{E}\left(E_{0}\right)$.

Кроме того, норма искажения композиции $G_{m, J} \circ g \circ \widetilde{G}_{I}^{-1}$ на интервале $\tilde{I}$ допускает равномерную оценку. Действительно,

$$
\eta\left(\widetilde{G}_{I} \circ g^{-1}, g(I)\right) \leqslant 2 C_{\eta}^{\prime}, \quad \eta\left(G_{m, J}, J\right) \leqslant C^{\prime} .
$$

Таким образом,

$$
\begin{aligned}
\eta\left(G_{m, J} \circ g \circ \widetilde{G}_{I}^{-1}, \tilde{I}\right) & =\sup _{K \subset \tilde{I}} \frac{\varkappa\left(G_{m, J} \circ g \circ \widetilde{G}_{I}^{-1} ; K\right)}{\left|\left(G_{m, J} \circ g \circ \widetilde{G}_{I}^{-1}\right)(K)\right|} \\
& \leqslant \sup _{K \subset \tilde{I}}\left(\frac{\varkappa\left(G_{m, J} ; g \circ \widetilde{G}_{I}^{-1}(K)\right)}{\left|\left(G_{m, J} \circ g \circ \widetilde{G}_{I}^{-1}\right)(K)\right|}+\frac{\varkappa\left(g \circ \widetilde{G}_{I}^{-1} ; K\right)}{\left|\left(G_{m, J} \circ g \circ \widetilde{G}_{I}^{-1}\right)(K)\right|}\right) \\
& \leqslant \eta\left(G_{m, J}, J\right)+\eta\left(\widetilde{G}_{I} \circ g^{-1} ; g(I)\right) \sup _{K \subset \tilde{I}} \frac{|K|}{\left|\left(G_{m, J} \circ g \circ \widetilde{G}_{I}^{-1}\right)(K)\right|} \\
& \leqslant C^{\prime}+2 C_{\eta}^{\prime} \frac{|\tilde{I}|}{\left|I^{\prime}\right|} \exp \left(\varkappa\left(G_{m, J} \circ g \circ \widetilde{G}_{I}^{-1} ; \tilde{I}\right)\right) \\
& \leqslant C^{\prime}+2 C_{\eta}^{\prime} \frac{1}{\varepsilon_{0}} \exp \left(C^{\prime}+2 C_{\eta}^{\prime} \frac{1}{\varepsilon_{0}}\right)=: C_{\eta}^{\prime \prime} .
\end{aligned}
$$


Значит, в силу леммы 4 отображение $G_{m, J} \circ g \circ \widetilde{G}_{I}^{-1}$ оказывается одним из конечного множества отображений

$$
\left\{\left.h\right|_{I}: h \in G, h(\tilde{I})=I^{\prime}, \eta(h, \tilde{I}) \leqslant C_{\eta}^{\prime \prime}\right\} .
$$

Итак, мы показали, что если длина интервала $I \in \mathcal{I}_{k}$ не превосходит $\varepsilon_{1}$, мы можем найти $m$ и $n$, такие, что соответствующие образы интервалов $I$ и $g(I)$ при растяжениях, $\tilde{I}=\widetilde{G}_{I}(I)=G_{n, I}(I)$ и $I^{\prime}=G_{m, J}(g(I))$, а также отображение $G_{m, J} \circ g \circ G_{n, I}^{-1}: \tilde{I} \rightarrow I^{\prime}$ принадлежат некоторым фиксированным конечным множествам.

Это завершает доказательство теоремы 2 при сделанном предположении.

Наконец, в общем случае для каждых двух принадлежащих одной орбите нерастягиваемых точек $x^{*}, \tilde{x}^{*}$ зафиксируем отображение $\tilde{h} \in G$, переводящее $x^{*}$ в $\tilde{x}^{*}$. Тогда сопряжение отображением $\tilde{h}$ соответствующего точке $\tilde{x}^{*}$ отображения $\tilde{g}_{+}$принадлежит $\operatorname{Stab}^{+}\left(x^{*}\right)$, и поэтому для некоторых $d_{1}, d_{2}$ в достаточно малой правой окрестности точки $x^{*}$ выполнено равенство $\tilde{h} \circ g_{+}^{d_{1}}=\tilde{g}_{+}^{d_{2}} \circ \tilde{h}$. Аналогично тому, как это делалось в замечании 6 , заменяя $\tilde{h}$ на его композицию с отрицательной степенью отображения $g_{+}$, можно считать, что это равенство выполнено и на всем прилегающем к $x_{*}$ интервале $I^{+}$.

Кроме того, если $G_{g\left(x^{*}\right), n_{0}}^{+}\left(g\left(x^{*}\right)\right)=\tilde{x}^{*}$, то $\tilde{h}^{-1} \circ G_{g\left(x^{*}\right), n_{0}}^{+} \circ g \in \operatorname{Stab}^{+}\left(x^{*}\right)$, откуда следует, что в достаточно малой правой окрестности точки $x^{*}$

$$
G_{g\left(x^{*}\right), n_{0}}^{+} \circ g=\tilde{h} \circ h_{i} \circ g_{+}^{l} .
$$

Аналогично рассуждению для случая одной нерастяжимой точки на орбите, взяв композицию отображения $(9)$ с $\tilde{g}_{+}^{d_{2} k}$ слева и с $g_{+}^{-\left(d_{1} k+l\right)}: I_{+} \rightarrow\left[x_{*}, y_{d_{1} k+l}\right]$ справа, при всех достаточно больших $k$ на $I_{+}$получаем

$$
\tilde{g}_{+}^{d_{2} k} \circ G_{g\left(x^{*}\right), n_{0}}^{+} \circ g \circ g_{+}^{-\left(d_{1} k+l\right)}=\tilde{g}_{+}^{d_{2} k} \circ\left(\tilde{h} \circ h_{i}\right) \circ g_{+}^{-d_{1} k}=\tilde{h} \circ h_{i} .
$$

Отсюда следует, что

$$
\left.g\right|_{\left[x^{*}, y_{d_{1} k+l}\right]}=\left(G_{g\left(x^{*}\right), n_{0}+d_{2} k}^{+}\right)^{-1} \circ\left(\tilde{h} \circ h_{i}\right) \circ G_{x^{*}, d_{1} k+l}^{+},
$$

и число возможных композиций $\tilde{h} \circ h_{i}$ конечно. После этого остальные рассуждения, приведенные для частного случая, повторяются дословно.

4.2. Доказательства вспомогательных утверждений. Прежде, чем доказывать предложение 3, докажем более слабое вспомогательное утверждение:

Лемма 7. Найдется такое $\varepsilon>0$, что если отображение $g \in G$ сохраняет некоторый интервал $I \in \mathcal{I} u$, кроме того, на этом интервале выполнена оценка $\left|g^{\prime}(x)-1\right|<\varepsilon$, то g сохраняет множсество $\Delta_{1}(I)$ концов интервалов разбиения первого уровня интервала $I$.

Доказательство. Если концы интервала $I$ не являются нерастяжимыми точками, то в этом случае множество разбивающих точек $\Delta_{1}(I)$ для разбиения первого уровня состоит из конечного множества точек конечной энергии, и мы можем положить $C_{I}=\max _{x \in \Delta_{1}(I)} E(x)$. С другой стороны, применение $g$ изменяет энергию точки $x$ в $g^{\prime}(x)$ раз (см. замечание 4$)$; поэтому при $\varepsilon<1 / 2$ образ $g(x)$ любой точки $x \in \Delta_{1}(I)$ обязан принадлежать энергетической области $\mathcal{E}\left(2 C_{I}\right)$. 
Но $\mathcal{E}\left(2 C_{I}\right)$ - конечное множество точек. Поэтому если диффеоморфизм $g$ не сохраняет хотя бы одну из точек множества $\Delta_{1}(I)$, мы получаем отделенную от 0 оценку на $\operatorname{dist}_{C^{1}}\left(\left.g\right|_{I}, \mathrm{id}\right)$. Действительно, пусть $a$ и $b$ - концы интервала $I$. Тогда по предположению $g(a)=a$ и $g(b)=b$. Если некоторая точка $x \in \Delta_{1}(I) \backslash \partial I$ не сохраняется при действии $g$, то по теореме Лагранжа о конечных приращениях $g^{\prime}(\xi)=|[a, g(x)]| /|[a, x]|$ для некоторого $\xi \in[a, x]$ и поэтому

$$
\left|g^{\prime}(\xi)-1\right| \geqslant \min _{x \in \Delta_{1}(I) \backslash \partial I, z \in \mathcal{E}\left(2 C_{I}\right) \backslash\{x, a\}}\left|\frac{|[a, z]|}{|[a, x]|}-1\right| .
$$

Выбрав $\varepsilon$ меньшим правой части в $(10)$ - положительной как минимум конечного числа положительных чисел, - мы получаем заключение леммы.

Пусть теперь один из концов интервала $I$ - нерастяжимая точка $x^{*} \in \operatorname{NE}(G)$ и $g_{+}-$соответствующее интервалу $I$ отображение. Обозначим через $J:=$ $g_{+}(I) \backslash I$ фундаментальную область и положим $J_{k}=g_{+}^{-k}(J)$.

Покажем, что если $\varepsilon>0$ достаточно мало, то из неравенства $\operatorname{dist}_{C^{1}}\left(\left.g\right|_{I}, \mathrm{id}\right)$ $<\varepsilon$ следует, что при всех $j \in \mathbb{N}$ отображение $g$ оставляет на месте точки из $g_{+}^{-j}\left(\Delta_{0}(J)\right)$. Поскольку $\bigcup_{j} g_{+}^{-j}\left(\Delta_{0}(J)\right)=\Delta_{1}(I)$, отсюда будет следовать заключение леммы.

Проведем доказательство по индукции. Для любого начального участка $j \leqslant j_{0}$ утверждение следует из тех же рассуждений, что и в первом случае, поскольку $\bigcup_{j \leqslant j_{0}} g_{+}^{-j}\left(\Delta_{0}(J)\right)$ - конечное множество точек конечной энергии. С другой стороны, $\lim _{j \rightarrow \infty}\left|J_{j}\right| /\left|J_{j+1}\right|=1$; следовательно,

существует $j_{0}$, такое, что $\left|J_{j}\right| /\left|J_{j+1}\right| \in[1 / 2,2]$ для любого $j \geqslant j_{0}$.

Если $\varepsilon<1 / 3$, то из сохранения отображением $g$ точек из $g_{+}^{-(j-1)}\left(\Delta_{0}(J)\right)$ и оценки $\left.g^{\prime}\right|_{I} \leqslant 1+\varepsilon$ следует, что $g\left(J_{j}\right) \subset J_{j} \cup J_{j+1}$. Отметим, что в силу предложения 1 все отображения $g_{+}^{-j}: J \cup J_{1} \rightarrow J_{j} \cup J_{j+1}$ имеют равномерно ограниченный некоторой константой $C_{\varkappa}$ коэффициент искажения (поскольку сумма длин образов не превосходит $2|I|+|J|)$. Поэтому для образа $g_{+}^{j}(g(x))$ точки $x \in g_{+}^{-j}\left(\Delta_{0}(J)\right)$ имеет место оценка

$$
\begin{aligned}
E\left(g_{+}^{j}(g(x))\right. & =\frac{E(x)}{\left(g_{+}^{j}\right)^{\prime}(g(x)) g^{\prime}(x)}=E\left(g_{+}^{j}(x)\right) \frac{\left(g_{+}^{j}\right)^{\prime}(x)}{\left(g_{+}^{j}\right)^{\prime}(g(x))} \frac{1}{g^{\prime}(x)} \\
& \leqslant\left(\max _{y \in \Delta_{0}(J)} E(y)\right) e^{C_{\varkappa}} \frac{1}{1-\varepsilon}=: C_{I}^{\prime} .
\end{aligned}
$$

Значит, образ $g_{+}^{j}(g(x))$ может принадлежать лишь конечному множеству $B=\mathcal{E}\left(C_{I}^{\prime}\right) \cap\left(J \cup J_{1}\right)$. Заметим, что в силу определения множества $B$ образ $g_{+}^{j}(x)$ также в нем содержится (соответствуя $\left.g=\mathrm{id}\right)$. Точки множества $B$ под действием $g_{+}^{-j}$ переходят в точку $x$ и конечное множество «потенциальных» образов $g(x)$. Но в силу ограниченности искажения все попарные расстояния между полученными точками сравнимы с длиной $J_{j}$ :

$$
\frac{\left|x_{1}-x_{2}\right|}{\left|J_{j}\right|} \geqslant e^{-C_{\varkappa}} \min _{z_{1} \neq z_{2}, z_{1}, z_{2} \in B} \frac{\left|z_{1}-z_{2}\right|}{|J|}>0 \quad \text { для любых } x_{1}, x_{2} \in g_{+}^{-j}(B) .
$$


Поэтому при $\varepsilon$, меньшем константы в правой части формулы (11) (обозначим ее через $\left.C_{d}\right)$, из сохранения отображением $g$ точек из $g^{-(j-1)}\left(\Delta_{0}(J)\right)(\mathrm{a}$, в частности, и граничной точки интервала $J_{j}$ ) путем рассуждений, аналогичных проведенным в первой части, получаем, что оно сохраняет точки из $g_{+}^{-j}\left(\Delta_{0}(J)\right)$.

Для завершения доказательства осталось заметить, что константа $C_{d}$ в правой части формулы $(11)$ не зависит от $\varepsilon$. Поэтому, выбрав $\varepsilon<\min \left(1 / 3, C_{d}\right)$ достаточно малым, чтобы гарантировать сохранение конечного множества $\bigcup_{j \leqslant j_{0}} g_{+}^{-j}\left(\Delta_{0}(J)\right)$, мы по индукции получаем сохранение всех точек из $\Delta_{1}(I)$.

Вторым вспомогательным утверждением, вместе с леммой 7 позволяющим доказать предложение 3, является

Лемма 8. Для любого $\varepsilon>0$ найдется $\varepsilon^{\prime}>0$, такое, что для любого $k \in \mathbb{N}$ и для любого интервала $I \in \mathcal{I}_{k}$ из того, что $g(I)=I u\left|g^{\prime}(x)-1\right|<\varepsilon^{\prime} \partial л я$ любого $x \in I$, следует, что для всех $x \in \widetilde{G}_{I}(I)$ выполняется неравенство $\mid\left(\widetilde{G}_{I}\right.$ ○ $\left.g \circ \widetilde{G}_{I}^{-1}\right)^{\prime}(x)-1 \mid<\varepsilon$.

Доказательство. Заметим, что для интервала $I$ из какого-либо разбиения $\mathcal{I}_{k}$ растяжения $F_{I}$ (см. определение 7$)$ и $\widetilde{G}_{I}$ различаются на применение одного из конечного множества отображений $\left\{g_{\tilde{I}} \mid \tilde{I} \in \mathcal{I}\right\}$. Поэтому в силу предложения 2, оценивающего норму искажения соответствующего отображения $F_{I}$, норма искажения отображения $\widetilde{G}_{I}: I \rightarrow \widetilde{G}_{I}(I)$ интервала $I \in \mathcal{I}_{k}$ любого уровня разбиения допускает равномерную оценку:

существует $\widetilde{C}_{\eta}$, такое, что $\eta\left(\widetilde{G}_{I^{\prime}}, I^{\prime}\right) \leqslant \widetilde{C}_{\eta}$ для любого $k^{\prime}$ и любого $I^{\prime} \in \mathcal{I}_{k^{\prime}}$.

Мы должны оценить, насколько отличается от 1 на $\widetilde{G}_{I}(I)$ производная композиции $\widetilde{G}_{I} \circ g \circ \widetilde{G}_{I}^{-1}$. Действительно, для $x \in \widetilde{G}_{I}(I), x=\widetilde{G}_{I}(z)$

$$
\left(\widetilde{G}_{I} \circ g \circ \widetilde{G}_{I}^{-1}\right)^{\prime}(x)=\frac{\widetilde{G}_{I}^{\prime}(g(z))}{\widetilde{G}_{I}^{\prime}(z)} g^{\prime}(z) \text {. }
$$

Второй сомножитель оценивается в силу предположения $\left|g^{\prime}\right|_{I}-1 \mid \leqslant \varepsilon^{\prime}$, a модуль логарифма первого не больше коэффициента искажения $\varkappa\left(\widetilde{G}_{I},[z, g(z)]\right)$, который, в свою очередь, оценивается так:

$$
\varkappa\left(\widetilde{G}_{I},[z, g(z)]\right) \leqslant \eta\left(\widetilde{G}_{I}, I\right)\left|\widetilde{G}_{I}([z, g(z)])\right| \leqslant \eta\left(\widetilde{G}_{I}, I\right) e^{\varkappa\left(\widetilde{G}_{I}, I\right)} \frac{|[z, g(z)]|}{|I|}\left|\widetilde{G}_{I}(I)\right| \text {. }
$$

Первые два сомножителя в правой части формулы (14) ограничены сверху в силу (12), последний не превосходит 1, а для третьего верна оценка

$$
\frac{|[z, g(z)]|}{|I|}=\left|\frac{|[a, g(z)]|-|[a, z]|}{|[a, z]|}\right|=\left|g^{\prime}(\xi)-1\right| \leqslant \varepsilon^{\prime},
$$

где $a$ - граничная точка интервала $I$, а точка $\xi \in[a, z]$ выбрана в соответствии с теоремой Лагранжа так, что $g^{\prime}(\xi)=|[a, g(z)]| /|[a, z]|$. Таким образом, задавшись достаточно малым $\varepsilon^{\prime}$, мы можем обеспечить малость логарифма первого сомножителя в правой части формулы (13), а тем самым, и выполнение неравенства

$$
\left|\left(\widetilde{G}_{I} \circ g \circ \widetilde{G}_{I}^{-1}\right)^{\prime}(x)-1\right| \leqslant \varepsilon \quad \text { для любого } x \in \widetilde{G}_{I}(I) .
$$


Доказательство предложения 3. Выберем $\varepsilon^{\prime}$, задаваемое леммой 8 по $\varepsilon$ из леммы 7. Докажем по индукции, что все интервалы $I^{\prime} \subset I, I^{\prime} \in \mathcal{I}_{k}, k \geqslant k_{0}$, сохраняются отображением $g$. Действительно, база $\left(k=k_{0}\right)$ является одним из условий предложения.

Шаг индукции обеспечивается леммой 8. А именно, если $I^{\prime} \subset I^{\prime \prime} \subseteq I, I^{\prime} \in$ $\mathcal{I}_{k+1}, I^{\prime \prime} \in \mathcal{I}_{k}$, то интервал $I^{\prime \prime}$ сохраняется отображением $g$ по предположению индукции и переводится отображением $\widetilde{G}_{I^{\prime \prime}}$ в некоторый марковский интервал $\widetilde{G}_{I^{\prime \prime}}\left(I^{\prime \prime}\right) \in \mathcal{I}$. Далее, из условия $\operatorname{dist}_{C^{1}}\left(\left.g\right|_{I}, \mathrm{id}\right) \leqslant \varepsilon^{\prime}$ и из леммы 8 следует, что

$$
\operatorname{dist}_{C^{1}}\left(\left.\widetilde{G}_{I^{\prime \prime}} \circ g \circ \widetilde{G}_{I^{\prime \prime}}^{-1}\right|_{\widetilde{G}_{I^{\prime \prime}}\left(I^{\prime \prime}\right)}, \mathrm{id}\right)<\varepsilon .
$$

Тогда по лемме 7 отображение $\widetilde{G}_{I^{\prime \prime}} \circ g \circ \widetilde{G}_{I^{\prime \prime}}^{-1}$ сохраняет $\Delta_{1}\left(\widetilde{G}_{I^{\prime \prime}}\left(I^{\prime \prime}\right)\right)$ и потому $g$ сохраняет

$$
\widetilde{G}_{I^{\prime \prime}}^{-1}\left(\Delta_{1}\left(\widetilde{G}_{I^{\prime \prime}}\left(I^{\prime \prime}\right)\right)\right)=\Delta_{k+1}\left(I^{\prime \prime}\right) .
$$

Таким образом, $g\left(I^{\prime}\right)=I^{\prime}$.

Так как $\bigcup_{k \geqslant k_{0}}\left(\Delta_{k}(I)\right)$ - плотное в $I$ множество, точки которого отображение $g$ оставляет на месте, то $\left.g\right|_{I}=\mathrm{id}$.

При доказательстве леммы 7 мы уже исследовали поведение энергии в окрестности нерастяжимой точки. Для доказательства леммы 5 нам придется ввести еще одно понятие, описывающее поведение энергии при таком приближении, и доказать с его помощью вспомогательное утвержение - предложение 5.

Пусть $x^{*}$ - нерастяжимая точка, обозначения $I^{+}, g_{+}, J, J_{k}-$ те же, что в абзаце перед замечанием 5 .

Предложение 4. Для любой точки $y \in G(\mathrm{NE}) \cap J$ существует предел

$$
E_{+}(y):=\lim _{k \rightarrow \infty} E\left(g_{+}^{-k}(y)\right)\left|J_{k}\right|
$$

и отношение $E_{+}(y) / E(y)$ отделено от нуля и равномерно ограничено по всем $y \in G(\mathrm{NE}) \cap J:$

существует $C_{+}$, такое, что $E(y) / C_{+} \leqslant E_{+}(y) \leqslant C_{+} E(y)$

$$
\text { для любого } y \in G(\mathrm{NE}) \cap J .
$$

Доказательство. Рассмотрим отношение двух допредельных величин в (15): пусть $k>l$, тогда

$$
\frac{E\left(g_{+}^{-l}(y)\right)\left|J_{l}\right|}{E\left(g_{+}^{-k}(y)\right)\left|J_{k}\right|}=\frac{E\left(g_{+}^{-l}(y)\right) / E\left(g_{+}^{-k}(y)\right)}{\left|J_{k}\right| /\left|J_{l}\right|} .
$$

Применив в числителе замечание 4 , связывающее энергию точки $x=g_{+}^{-l}(y)$ с энергией ее образа $g_{+}^{-(k-l)}(x)=g_{+}^{-k}(y)$, а в знаменателе теорему Лагранжа, получаем

$$
\frac{E\left(g_{+}^{-l}(y)\right)\left|J_{l}\right|}{E\left(g_{+}^{-k}(y)\right)\left|J_{k}\right|}=\frac{\left(g_{+}^{-(k-l)}\right)^{\prime}\left(g_{+}^{-l}(y)\right)}{\left(g_{+}^{-(k-l)}\right)^{\prime}(\xi)},
$$

где $\xi \in J_{l}$ и $\left(g_{+}^{-(k-l)}\right)^{\prime}(\xi)=\left|J_{k}\right| /\left|J_{l}\right|$. Наконец, логарифмируя, получаем

$$
\left|\ln \frac{E\left(g_{+}^{-l}(y)\right)\left|J_{l}\right|}{E\left(g_{+}^{-k}(y)\right)\left|J_{k}\right|}\right|=\left|\ln \frac{\left(g_{+}^{-(k-l)}\right)^{\prime}\left(g_{+}^{-l}(y)\right)}{\left(g_{+}^{-(k-l)}\right)^{\prime}(\xi)}\right| \leqslant \varkappa\left(g_{+}^{-(k-l)}, J_{l}\right) .
$$


Поскольку образы фундаментальной области $J_{k}=g_{+}^{-k}(J)$ при итерациях $g_{+}^{-1}$ дизъюнктны, сумма их длин сходится, и потому коэффициент искажения $\varkappa\left(g_{+}^{-k}, J\right)$ равномерно по $k$ ограничен (см. предложение 1$)$. Более того, коэффициенты искажения $\varkappa\left(g_{+}^{-(k-l)}, J_{l}\right), k>l$, стремятся к нулю при $l \rightarrow \infty$, поскольку соответствующая сумма длин оценивается «хвостом» сходящегося ряда. Таким образом, из (17) следует, что логарифмы $\ln \left(E\left(g_{+}^{-k}(y)\right)\left|J_{k}\right|\right)$ образуют последовательность Коши и потому сходятся.

Наконец, переходя в (17) к пределу при $l=0, k \rightarrow \infty$ и используя лемму 1 , мы видим, что для константы $C_{+}$достаточно потребовать, чтобы выполнялось неравенство $\ln C_{+} \geqslant \sup _{k} \varkappa\left(g^{-k} ; J\right)+|\ln | J||$.

Следствие 3. Для любого $C$ множество $\mathcal{E}_{+}(C)=\left\{y \in J \mid E_{+}(y) \leqslant C\right\}$ конечно. В частности, множсество значений функции $E_{+}$дискретно.

Пусть $k(\cdot): I^{+} \rightarrow \mathbb{N}$ - номер образа фундаментальной области, содержащего данную точку: $J_{k(x)} \ni x$ для любого $x \in I^{+}$. Тогда имеет место следующее

Предложение 5. Для любого $g \in G$, такого, что $g\left(x^{*}\right)=x^{*}$,

$$
\frac{\left|J_{k(x)}\right|}{\left|J_{k(g(x))}\right|} \underset{x \rightarrow x^{*}, x \in I^{+}}{\longrightarrow} 1 \text {. }
$$

Доказательство. Продолжим функцию $E_{+}$на весь интервал $I^{+} g_{+}$-инвариантным образом: для $x \in J_{j}$ положим $E_{+}(x)=E_{+}\left(g_{+}^{-j}(x)\right)$. Тогда при $x \rightarrow x^{*}$, $x \in G(\mathrm{NE}) \cap I^{+}$,

$$
\begin{aligned}
\frac{E_{+}(x)}{E(x)\left|J_{k(x)}\right|} & \rightarrow 1, \\
\frac{E_{+}(g(x))}{E(g(x)) \mid J_{k(g(x))}} & \rightarrow 1 .
\end{aligned}
$$

Рассмотрим последовательность $x_{l}:=g_{+}^{-l}\left(x_{0}\right)$, где точка $x_{0} \in J \cap G(\mathrm{NE})$ выбрана как точка, в которой функция $E_{+}$принимает наименьшее значение. (Такая найдется, поскольку в силу следствия 3 мы можем ограничить выбор конечным набором точек $\mathcal{E}_{+}\left(E_{+}(z)\right)$ для произвольного $\left.z.\right)$

Докажем для начала более слабую версию (18), а именно,

$$
\liminf _{l \rightarrow \infty} \frac{\left|J_{k\left(g\left(x_{l}\right)\right)}\right|}{\left|J_{l}\right|} \geqslant 1 \text {. }
$$

Разделив (19) на (20) и подставив $x=x_{l}$ (для которого, таким образом, $\left.k\left(x_{l}\right)=l\right)$, получаем

$$
\frac{E_{+}\left(x_{l}\right)}{E_{+}\left(g\left(x_{l}\right)\right)} \frac{E\left(g\left(x_{l}\right)\right)}{E\left(x_{l}\right)} \frac{\left|J_{k\left(g\left(x_{l}\right)\right)}\right|}{\left|J_{l}\right|} \rightarrow 1, \quad l \rightarrow \infty .
$$

Первый сомножитель в $(21)$ не превосходит 1 в силу выбора $x_{0}$, второй равен $g^{\prime}\left(x_{l}\right)$ и потому стремится к 1 , а следовательно,

$$
\liminf _{l \rightarrow \infty} \frac{\mid J_{k\left(g\left(x_{l}\right)\right) \mid}}{\left|J_{l}\right|} \geqslant 1 \text {. }
$$


Далее, при $\left|J_{k\left(g\left(x_{l}\right)\right)}\right| /\left|J_{l}\right| \geqslant 2 / 3$ и при достаточно близких к 1 отношениях длин пяти интервалов $J_{k\left(g\left(x_{l}\right)\right)-2}, J_{k\left(g\left(x_{l}\right)\right)-1}, \ldots, J_{k\left(g\left(x_{l}\right)\right)+2}$ образ $g\left(J_{l}\right)$ покрывается объединением этих пяти интервалов и, следовательно,

$$
\left|k(g(y))-k\left(g\left(x_{l}\right)\right)\right| \leqslant 2 \quad \text { для любого } y \in J_{l} .
$$

Из (22) и замечания 5 тогда имеем

$$
\liminf _{y \rightarrow x^{*}+0} \frac{\left|J_{k(g(y))}\right|}{\left|J_{k(y)}\right|}=\liminf _{y \rightarrow x^{*}+0} \frac{\left|J_{k(g(y))}\right|}{\left|J_{k\left(g\left(x_{k(y)}\right)\right.}\right|} \cdot \frac{\left|J_{k\left(g\left(x_{k(y)}\right)\right.}\right|}{\left|J_{k(y)}\right|} \geqslant 1 .
$$

Наконец, то же неравенство выполнено и для отображения $g^{-1}$; объединяя их, получаем искомое утверждение.

Доказательство леммы 5. Как и в доказательстве предложения 5, распространим функцию $E_{+}(y)$ с $J$ на интервал $I^{+} g_{+}$-инвариантным образом. Положим теперь $\widetilde{\mathcal{E}}_{+}(C):=\left\{x \in I^{+} \cup J \mid E_{+}(x) \leqslant C\right\}$. Множество значений функции $E_{+}$дискретно, но, с другой стороны, из предложения 5 следует, что

$$
\lim _{x \rightarrow x^{*}+0} \frac{E_{+}(g(x))}{E_{+}(x)}=1 .
$$

Поэтому для любого фиксированного значения $C$ в достаточно малой правой окрестности $U_{+}=\left(x^{*}, x^{*}+\delta\right)$

$$
E_{+}(x) \leqslant C \Longleftrightarrow E_{+}(g(x)) \leqslant C .
$$

Положим $C:=\max _{y \in \Delta_{0}(J)} E_{+}(y)$, и пусть $z_{1}, \ldots, z_{N}, z_{N+1}-$ пронумерованные в порядке приближения к $x^{*}$ точки из $J$, принадлежащие $\mathcal{E}_{+}(C)$ (тем самым, $z_{1}$ и $z_{N+1}$ оказываются концами интервала $\left.J\right)$ и $z_{j+N k}=g_{+}^{-k}\left(z_{j}\right)$ для $z_{j} \in J$. Тогда все точки $\left\{z_{j}\right\}-$ это в точности точки из $\widetilde{\mathcal{E}}_{+}(C)$, пронумерованные в порядке приближения к $x^{*}$. Заметим, что в силу (23) применение $g$ в некоторой окрестности точки $x^{*}$ переводит это множество в себя, а тем самым, просто сдвигает нумерацию:

существуют $n_{0} \in \mathbb{N}, d=d(g) \in \mathbb{Z}$, такие, что $g\left(z_{n}\right)=z_{n+d}$ для любого $n \geqslant n_{0}$.

Рассмотрим отображение $g \mapsto d(g)$. Оно является гомоморфизмом из $\operatorname{Stab}^{+}\left(x^{*}\right)$ в $\mathbb{Z}$. Действительно, $d\left(g_{1} \circ g_{2}\right)=d\left(g_{1}\right)+d\left(g_{2}\right)$, поскольку последовательное применение $g_{2}$ и $g_{1}$ сдвигает нумерацию сначала на $d\left(g_{2}\right)$, а потом на $d\left(g_{1}\right)$ в некоторой малой окрестности нерастяжимой точки.

Более того, этот гомоморфизм инъективен как отображение из группы правых ростков. Действительно, если $d(g)=0$, то $g$ оставляет на месте все достаточно близкие к $x^{*}$ точки из $\Delta_{1}\left(I^{+}\right)$. Поэтому интервалы первого уровня разбиения, лежащие в достаточно малой правой окрестности точки $x^{*}$, оказываются $g$-инвариантными. С другой стороны, поскольку $g^{\prime}\left(x^{*}\right)=1$, диффеоморфизм $g$ является $C^{1}$-близким к тождественному в некоторой малой окрестности точки $x^{*}$. Тогда в силу предложения 3 найдется правая окрестность нерастяжимой точки, в которой $g=$ id на каждом из интервалов разбиения первого уровня. Таким образом, и просто в некоторой правой окрестности нерастяжимой точки $g=\mathrm{id}$.

В качестве отображений $h_{i}$ теперь можно выбрать по одному отображению из класса смежности группы $\operatorname{Stab}^{+}\left(x^{*}\right)$ по $\left\langle g^{+}\right\rangle$: порожденная числом $d\left(g^{+}\right)$ 
группа имеет конечный индекс в $\mathbb{Z}$, и потому число таких классов смежности конечно.

Наконец, поскольку гомоморфизм в абелеву группу $\mathbb{Z}$ из группы правых ростков инъективен, любые два отображения из стабилизатора (в частности, $g_{+}$и $\left.h_{i}\right)$ коммутируют в некоторой окрестности нерастяжимой точки.

Доказательство леммы 6. Сразу отметим, что третье заключение будет являться следствием второго: поскольку множество $\mathcal{E}\left(E_{0}\right)$ конечно, попарные расстояния между его точками отделены от нуля.

Покажем для начала, что выполнено первое заключение леммы. А именно, рассмотрим интервал $I^{\prime}:=F_{J}(J)$. В случае, если $F_{J}=G_{J}$, утверждение автоматически следует из предложения 2. В противном случае интервал $I^{\prime}$ содержится в прилегающем к некоторой нерастяжимой точке $x^{*}$ интервале разбиения $I^{+}$и пересекает $\Delta_{1}\left(I^{+}\right)$. Пусть для определенности $I^{+}-$правый из соответствующих интервалов; обозначим концы интервала $I^{\prime}$ через $a$ и $b$ и воспользуемся стандартными обозначениями $g_{+}, J^{+}, J_{k}^{+}$.

Тогда $G_{J}=g_{+}^{k} \circ F_{J}$, где $b \in J_{k}^{+}$. Покажем, что найдутся $N_{1}, N_{2}$, не зависящие от $I$ или $g$, для которых либо $k \leqslant N_{1}$, либо $g_{+}^{-N_{2}}(b)<a$. В обоих случаях норма искажения отображения $\left.g_{+}^{k}\right|_{I^{\prime}}$ оказывается проконтролированной автоматически в силу оценки на сумму длин образов и предложения 1.

Для этого заметим сначала, что коэффициент искажения композиции $g \circ F_{J}^{-1}: I^{\prime} \rightarrow I$ можно оценить так:

$$
\varkappa\left(g \circ F_{J}^{-1} ; I^{\prime}\right) \leqslant \varkappa(g ; J)+\varkappa\left(F_{J}^{-1} ; I^{\prime}\right) \leqslant C|g(J)|+C_{\eta}\left|I^{\prime}\right| \leqslant C+C_{\eta} .
$$

Поэтому если энергия некоторой точки $x \in I^{\prime}$ не более чем вдвое превосходит энергию концевой точки $b$, то энергия ее образа $g \circ F_{J}^{-1}(x)$ не больше, чем $e^{C+C_{\eta}} 2 \max _{x \in \Delta_{0}} E(x)=: C_{2}$.

Но таких точек не более, чем $\# \mathcal{E}\left(C_{2}\right)$, так что если $I^{\prime}$ не накрывается объединением $N_{2}=\# \mathcal{E}\left(C_{2}\right)+1$ фундаментальных областей, то одна из производных $\left(g_{+}^{-1}\right)^{\prime}(b),\left(g_{+}^{-2}\right)^{\prime}(b), \ldots,\left(g_{+}^{-N_{2}}\right)^{\prime}(b)$ не больше $1 / 2$. С другой стороны, каждая из этих производных стремится к единице, когда $b \rightarrow x^{*}+0$; поэтому такое условие отделяет $b$ от $x^{*}$. Таким образом, если $I^{\prime}$ не накрывается объединением $N_{2}$ фундаментальных областей, то число итераций отображения $g_{+}$до того, как точка $b$ покинет интервал $I^{+}$, оказывается ограниченным некоторой константой $N_{1}$.

Следовательно, норма искажения $\eta\left(G_{J} ; J\right)$ ограничена некоторой константой $C^{\prime}$. Это доказывает первое из заключений леммы.

Заметим теперь, что (аналогично (24)) для коэффициента искажения композиционного частного $g \circ G_{J}^{-1}$ выполнена оценка

$$
\varkappa\left(g \circ G_{J}^{-1} ; G_{J}(J)\right) \leqslant C|g(J)|+C^{\prime}\left|G_{J}(J)\right| \leqslant C+C^{\prime} .
$$

По определению $G_{J}$ образ $G_{J}(J)$ пересекает множество граничных точек $\Delta_{0}$ разбиения $\mathcal{I}$. Пусть $x \in G_{J}(J) \cap \Delta_{0}$. Тогда, сравнивая энергию любой из концевых точек $y \in \partial\left(G_{J}(J)\right)$, точки $x$ и их $g \circ G_{J}^{-1}$-образов, имеем

$$
\frac{E(y)}{E(x)}=\frac{E\left(g \circ G_{J}^{-1}(y)\right)}{E\left(g \circ F_{J}^{-1}(x)\right)} \frac{\left(g \circ G_{J}^{-1}\right)^{\prime}(y)}{\left(g \circ G_{J}^{-1}\right)^{\prime}(x)} .
$$


Второй сомножитель в правой части не превосходит $e^{C+C^{\prime}}$, точки $x$ и $g \circ G_{J}^{-1}(y)$ принадлежат множеству $\Delta_{0}$ граничных точек, а потому их энергия не превосходит $C_{0}:=\max _{x \in \Delta_{0}} E(x)$. Наконец, $E\left(g \circ G_{J}^{-1}(x)\right) \geqslant 1$, откуда получаем требуемое

$$
E(y) \leqslant \frac{C_{0}^{2}}{1} e^{C+C^{\prime}}=: E_{1} .
$$

Доказательство леммы 4. Рассмотрим множество $X$ таких отображений с расстоянием $d\left(g_{1}, g_{2}\right)=\operatorname{dist}_{C^{1}(I)}\left(g_{1}^{-1} \circ g_{2}, \mathrm{id}\right)$.

С одной стороны, оценка на норму искажения, соответствующая оценке на вторую производную, в силу теоремы Арцела-Асколи влечет за собой предкомпактность пространства $(X, d)$.

С другой - из предложения 3 следует, что для всякого элемента множества $X$ в его $\varepsilon^{\prime}$-окрестности других точек из $X$ нет. Значит, $X$ конечно.

\section{§5. Доказательство следствия 1}

Доказательство следствия 1. Достаточно показать, что любое конечное подмножество $\left\{x_{1}, \ldots, x_{l}\right\} \subset G(x)$ разбивается на не более чем $N$ классов $\mathcal{R}$-эквивалентности. Для этого представим точки такого подмножества как $g_{(1)}(x), \ldots, g_{(l)}(x)$, где отображения $g_{(j)}$ принадлежат $G$. По теореме 2 в некоторой окрестности $U$ точки $x$ все эти отображения могут быть представлены KaK

$$
\left.g_{(i)}\right|_{U}=G_{n_{j}, g_{(j)}(x)}^{-1} \circ h_{i_{j}} \circ G_{m, x}
$$

с $m$, определяемым окрестностью $U$ и, значит, не зависящим от $j$. Отсюда следует, что

$$
R^{n_{j}}\left(x_{j}\right)=G_{n_{j}, g_{(j)}(x)}\left(g_{(i)}(x)\right)=h_{i_{j}}\left(R^{m}(x)\right)
$$

и наше подмножество $\left\{x_{1}, \ldots, x_{l}\right\}$ вложено в объединение $N$ классов $\mathcal{R}$-эквивалентности точек $h_{1}\left(R^{m}(x)\right), \ldots, h_{N}\left(R^{m}(x)\right)$.

\section{ЛиТЕРАТУРА}

[1] R. Bowen, Invariant measures for Markov maps on the interval, Comm. Math. Phys., 69:1 (1979), 1-17.

[2] R. Bowen, C. Series, Markov maps associated with Fuchsian groups, Pub. Math. IHES, 50:1 (1979), 153-170.

[3] J. Cantwell, L. Conlon, Foliations and subshifts, Tôhoku Math. J., 40:2 (1988), 165187.

[4] B. Deroin, V. Kleptsyn, A. Navas, On the question of ergodicity for minimal group actions on the circle, Mosc. Math. J., 9:2 (2009), 263-303.

[5] B. Deroin, V. Kleptsyn, A. Navas, Sur la dynamique unidimensionnelle en régularité intermédiaire, Acta Math., 199:2 (2007), 199-262.

[6] É. Ghys, V. Sergiescu, Sur un groupe remarquable de difféomorphismes du cercle, Comment. Math. Helv., 62:2 (1987), 185-239.

[7] Y. Guivarc'h, Y. Le Jan, Asymptotic winding of the geodesic flow on modular surfaces and continued fractions, Ann. Sc. Ec. Norm. Sup. (4), 26:1 (1993), 23-50.

[8] Y. Guivarc'h, C. R. E. Raja, Recurrence and ergodicity of random walks on linear groups and on homogeneous spaces, http://arxiv.org/abs/0908.0637.

[9] M.-R. Herman, Sur la conjugaison différentiable des difféomorphismes du cercle à des rotations, Publ. Math. de l'IHES, 49 (1979), 5-234. 
[10] S. Hurder, Exceptional minimal sets and the Godbillon-Vey class, Annales de l'Institut Fourier (Grenoble) (to appear).

[11] T. Inoue, Ratio ergodic theorems for maps with indifferent fixed points, Ergodic Theory Dynam. Systems, 17:3 (1997), 625-642.

[12] А. Б. Каток, Б. Хасселблат, Введение в теорию динамических систем с обзором последних достижений, МЦНМО, М., 2005.

[13] В. А. Клепцын, Д. А. Филимонов, Показатели Ляпунова и другие свойства N-груnп, Труды ММO, 73:1 (2012).

[14] R. Mañé, Introdução à teoria ergódica, Projeto Euclides, 1983; English transl.:Ergodic Theory and Differentiable Dynamics, Springer-Verlag, Berlin, 1987.

[15] A. Navas, Sur les groupes de difféomorphismes du cercle engendrés par des éléments proches des rotations, Enseign. Math., 50:1-2 (2004), 29-68.

[16] A. Navas, Grupos de difeomorfismos del círculo, Ensaios Matemáticos, Braz. Math. Society, 2007; English transl.:Groups of circle diffeomorphisms, http://arxiv.org/ abs/math/0607481.

[17] M. Shub, D. Sullivan, Expanding endomorphisms of the circle revisited, Ergodic Theory Dynam. Systems, 5:2 (1985), 285-289.

CNRS, Institut de Recherche Mathématique de Rennes e-mail: victor.kleptsyn@univ-rennes1.fr

Поступило в редакцию

25 июня 2010 г.

Московский физико-технический институт (МФТИ)

Московский государственный университет путей сообщения (МИИТ)

e-mail: mityafil@gmail.com 